\title{
VARIACIÓN TEMPORAL Y ESPACIAL EN LA ESTRUCTURA DE LA COMUNIDAD DE PEQUEÑOS MAMÍFEROS EN UN BOSQUE TROPICAL SECO
}

\author{
YOLANDA, DOMÍNGUEZ-CASTELLANOS ${ }^{1}$ Y GERARDOCEBALLOS $^{1}$ \\ ${ }^{1}$ Laboratorio de Ecología y Conservación de Fauna Silvestre, Instituto de Ecología, UNAM. \\ Circuito Ext. s/n anexo al Jardín Botánico, Cd. Universitaria México, D.F. 04510. Teléfono y \\ fax: 56229004 correo electrónico: yodoca@ecologia.unam.mx
}

Resumen: La estructura de una comunidad biológica está conformada por atributos como la composición, la riqueza y la abundancia de las especies, e influenciada por los cambios temporales y espaciales, que a su vez, se refleja en la composición y abundancia de especies de dicha comunidad. El objetivo fue evaluar la variabilidad temporal y espacial de la comunidad de pequeños mamíferos en la selva seca de Chamela, Jalisco, considerando los dos tipos de vegetación predominantes (selva baja y selva mediana). El muestreo abarcó 18 años y la información generada se compiló en una base de datos, en la que se incluyen los registros de precipitación de los mismos periodos. Con los datos obtenidos se concluye que la abundancia anual de especies varió año con año, registrándose 13 especies de pequeños mamíferos en el área (Tlacuatzin canescens, Megasorex gigas, Spilogale pygmaea, Liomys pictus, Oryzomys melanotis, O. couesi, Nyctomys sumichrasti, Reithrodontomys fulvescens, Osgoodomys banderanus, Peromyscus perfulvus, Baiomys musculus, Sigmodon mascotensis y Xenomys nelsoni). A pesar de que la estructura y cambios temporales de la vegetación modificaron de forma distinta a cada tipo de selva, la composición fue igual para ambas, no obstante la selva mediana es más rica y diversa en especies que la selva baja. Esta estructura de la comunidad de pequeños mamíferos mostró una variación a largo plazo tanto en composición como en riqueza. Por lo que las características de la región de Chamela, permiten la coexistencia de las especies haciendo un uso diferencial del hábitat.

Palabras clave: Pequeños mamíferos, comunidad, selvas secas, Jalisco, México.

\begin{abstract}
The structure of a biological community consists of attributes such as composition, richness and abundance of species, and influenced by the temporal and spatial changes, which in turn is reflected in the composition and abundance of species of that community. The objective was to evaluate the temporal and spatial variability of the community of small mammals in the dry forest of Chamela, Jalisco, considering the two predominant types of vegetation (lowland and tropical forest). The sample comprised 18 and the information generated is compiled in a database, which includes the precipitation records the same periods. The data obtained showed that the annual abundance of species varied from year to year, recorded 13 species of small mammals in the area (Tlacuatzin canescens, Megasorex gigas, Spilogale pygmaea, Liomys pictus, Oryzomys melanotis, O. couesi, Nyctomys sumichrasti,
\end{abstract}


Reithrodontomys fulvescens, Osgoodomys banderanus, Peromyscus perfulvus, Baiomys musculus, Xenomys nelsoni and Sigmodon mascotensis). Although the structure and temporal changes of vegetation changed differently for each type of forest, the composition was the same for both, despite the tropical forest is richer and more diverse in the lowland species. The community structure of small mammals showed a long-term variation in composition and in wealth. So that the characteristics of the region of Chamela allow coexistence of species making a differential use habitat.

Key words: small mammals, community, dry forest, Jalisco, Mexico.

\section{INTRODUCCIÓN}

Una comunidad ecológica, está conformada por atributos como la composición, la riqueza y la abundancia de las especies, que a su vez son afectadas por las variaciones temporales y espaciales originadas por el efecto de las condiciones ambientales (Brady y Slade, 2001; Diffendorfer et al., 1996; Krebs, 1985; Lekve et al., 2002). Particularmente, en los ambientes terrestres se presenta una heterogeneidad ambiental generada principalmente por estos cambios (Brown y Heske, 1990; Brown et al., 2001) y la variación generada en las condiciones abióticas limita la distribución de las poblaciones animales, afectando directamente sus ciclos de vida e indirectamente los tipos de estructura, fenología y productividad de la vegetación (Watkins y Wilson, 1994; Wolda, 1988).

En los bosques tropicales deciduos surgen cambios tanto en la fenología como en la disponibilidad de alimento, especialmente en la temporada de lluvias, ya que la cantidad y patrón de lluvia determinan que la reproducción y los movimientos locales de las especies sean estacionales, a diferencia de lo que ocurre en los bosques lluviosos (Bullock y SolísMagallanes, 1990; Ceballos, 1989, 1990; M'Closkey, 1972; Owen, 1990; Trejo, 2010; Viveiros, 2003; Wells et al., 2004).

Se ha observado que en los años en que el Fenómeno de El Niño ocurre, en intervalos de 2 a 7 años, los patrones climáticos modifican el sitio, es decir, se muestran intensas sequías o abundantes lluvias (Brown y Ernest, 2002; Lima et al., 1999; Milstead et al., 2007; Stenseth et al., 2002).

Es así que la estacionalidad para los bosques templados, a diferencia de los tropicales, es originada por la temperatura, permitiendo que los ritmos temporales de plantas y los ciclos de vida animal sean más estables y tengan un flujo constante dentro de la comunidad, especializándose las especies en varios nichos (Owen, 1990). En cambio, en los bosques tropicales, la precipitación es importante porque los animales presentan movimientos locales o regionales, ya que cambian sus patrones de actividad, de dieta, de acumulación de recursos y sus adaptaciones fisiológicas para la obtención de agua (Ceballos, 1995; Townsend et al., 2000). 
En México, en la vertiente del Pacífico, las selvas bajas o selvas secas o bosques tropicales deciduos se localiza principalmente en las laderas, mientras que las selvas medianas o bosques subperennifolios se asocian a los cursos de aguas temporales o permanentes que corren a lo largo de cañadas. Ambos sitios (laderas y cañadas) están sometidos a las mismas condiciones climáticas, sin embargo, la diferencia está dada por su estructura vegetal, composición taxonómica, productividad, fenología y topografía, permitiendo que la precipitación se acumule sólo en los arroyos profundos (Lott et al., 1987; Trejo, 2010).

La variabilidad tanto en la selva baja como de la selva mediana esta dada no sólo por un incremento en el gradiente de la complejidad estructural espacial, sino además por un decremento temporalestacional generado por la cantidad de lluvia, en la selva mediana. Lo que provee de un ambiente con una heterogeneidad ambiental en los que se pueden comparar los procesos de las poblaciones y de la comunidad (Ceballos y Martínez, 2010). En este trabajo se describe cómo en 18 años la heterogeneidad del hábitat, en dos tipos de selvas con características diferentes y con las mismas condiciones climáticas, tuvo una influencia en la comunidad de los pequeños mamíferos; modificando espacial y temporalmente la estructura de la comunidad de las especies, considerando la abundancia, la riqueza y la composición. Asimismo, se determina los cambios en la estacionalidad climática tanto en periodos cortos de tiempo como en largos provocaron variaciones en la estructura de dicha comunidad.

Además de que a nivel espacial y temporal, la composición y la riqueza de especies se ve influenciada por la disponibilidad de recursos. Y finalmente, se registra el efecto diferencial en la variación en los patrones estacionales y anuales de la estructura de la comunidad, al compararse los registros de los años secos y de los años lluviosos.

\section{MÉTODOS}

\section{Área de estudio}

El estudio se desarrolló en la Estación de Biología Chamela, que forma parte de la Reserva de la Biosfera Chamela-Cuixmala, en el estado de Jalisco. La estación tiene un área de 3,370 hectáreas y se localiza a los $19^{\circ} 30^{\prime} \mathrm{Ny} 105^{\circ} 03^{\prime} \mathrm{O}$ (Figura 1; Bullock, 1986; Ceballos y García, 2010; Ceballos y Miranda, 2000; Lott et al., 1987). La altitud en el área varia de 20 a 500 m.s.n.m., y su topografía consiste de laderas, cañadas y cursos de agua temporales. El clima se caracteriza por una marcada estacionalidad, y a lo largo del año se presentan tres temporadas: lluvias (julio a octubre), post-lluvias (noviembre a febrero) y secas (marzo a junio). La temperatura promedio es de $24.9^{\circ} \mathrm{C}$ y la precipitación promedio mensual es de 748 mm (Bullock, 1986; Castellanos et al., 1989; Ceballos y García, 2010; Ceballos et al., 1999; Lott y Atkinson, 2010). Los tipos de vegetación predominante son: la selva baja caducifolia, que se encuentra 
distribuida en lomeríos; con un estrato arbóreo que alcanza alturas entre los 4 y $15 \mathrm{mts}$, un estrato arbustivo que se presenta sobre todo en las laderas y un estrato herbáceo que se desarrolla solamente durante la temporada de lluvias. El otro tipo, la selva mediana subperennifolia se encuentra en las cercanías de los arroyos permanentes y de temporal, presenta dos estratos arbóreos: uno hasta 15 mts y otro entre 25 y 40 mts (Ceballos y Miranda, 1986, 2000; Lott et al., 1987; Martínez-Yrizar et al., 1996). Además cuenta con los estratos arbustivo, herbáceo y enredaderas leñosas, los cuales difieren de los presentes en la selva baja (Bullock y SolísMagallanes, 1990; Lott, 1985).

\section{Muestreo}

Se utilizaron seis cuadrantes de media hectárea, 4 en la selva baja con 64 trampas tipo Sherman y 2 cuadrantes en la selva mediana de 95 y 85 trampas, respectivamente. Las trampas tenían una disposición espacial de 8 × 8 metros de separación. Se colocaron sólo en suelo para la selva baja y tanto en suelo como en árboles para la selva mediana. Las trampas se cebaron con una mezcla de crema de cacahuate, avena y vainilla. Se dejaron en los sitios por tres noches consecutivas durante la fase de la luna nueva. A los ejemplares capturados se les tomó datos de la fecha y sitio de captura, especie, peso, sexo y condición

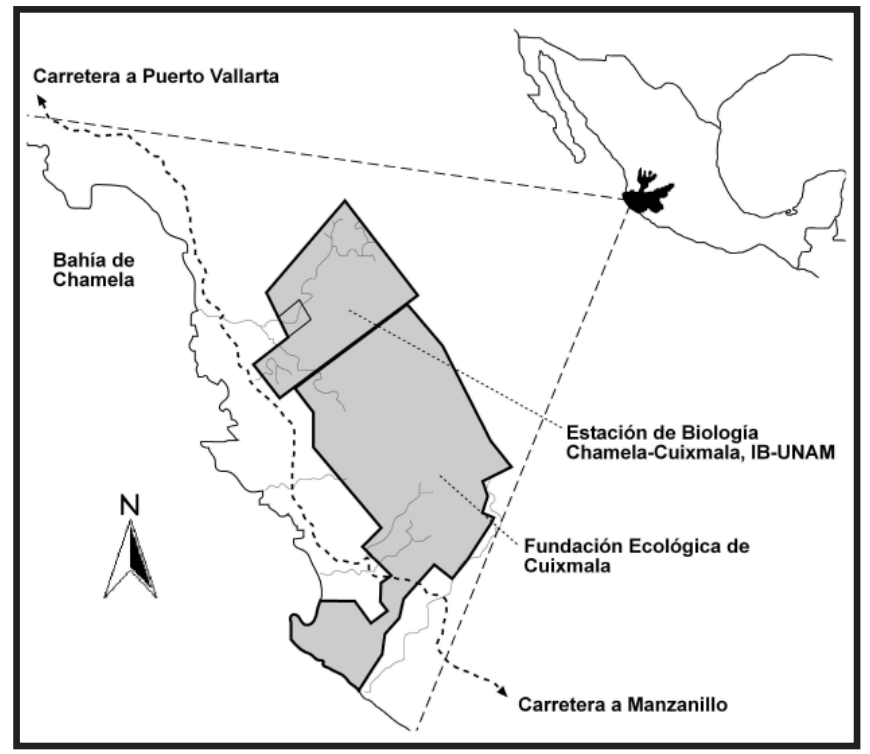

Figura 1. Estación de Biología Chamela, Jalisco en la Reserva de la Biosfera Chamela, Cuixmala. 
reproductiva: preñadas o lactantes en el caso de las hembras y con testículos semiescrotados o escrotados en el caso de los machos. Finalmente, los ejemplares colectados se marcaron con la técnica de ectomización de falanges, siguiendo un número progresivo para cada una de las especies (Mendoza, 1997).

\section{Variación temporal y espacial}

Para conocer los patrones temporales y espaciales de la comunidad de los pequeños mamíferos, se formó una base de datos de 18 años (1990 a 2007), la cual contiene registros de datos mensuales (1990 a 1994) y bimensuales (1995 a 2007). Asimismo, se contó con los datos de los registros de precipitación para la estación más cercana durante estos años (Estación Meteorológica de Chamela).

Se calcularon los esfuerzos de muestreo y los éxitos de captura para cada uno de los años. Para determinar si los datos presentaban una distribución normal se realizó un análisis preliminar con la prueba de Kolmogorov-Smirnov. Posteriormente se compararon las abundancias y el número de especies por tipo de vegetación mediante un análisis de varianza y, al registrarse diferencias significativas, se utilizó la prueba de comparación múltiple de Tukey (HSD) para definir cual fue el grupo que mostró dicha diferencia (Kuypers, 2001).

\section{Diversidad y riqueza de especies}

Para calcular la diversidad de las especies de roedores de cada tipo de vegetación, se utilizó el Índice de Diversidad de Shannon-Wiener (H'), el cual asume que los individuos fueron muestreados al azar y que todas las especies estuvieron representadas en la muestra, además, de obtener la riqueza de especies (S) y la equitatividad (J') (Neil McAleece et al, 1997; Magurran, 1988).

También se calculó la diversidad máxima (H’ máx), que representa la diversidad de todas las especies de la comunidad con igual abundancia. La equitatividad (J') indica como están distribuidos los individuos entre las especies y representa el valor entre la diversidad observada y la diversidad máxima (Krebs, 1985). Para probar si las diversidades de las dos poblaciones son iguales se utilizó la prueba de $t$ de Hutchinson (Magurran, 1988; Zar, 1996).

Se realizaron correlaciones de Spearman $\left(r^{2}\right)$ para observar que variable era la más afectada por la precipitación pluvial. Se consideraron la precipitación acumulada y la precipitación promedio con la abundancia absoluta, la riqueza, la diversidad y la diversidad máxima. Se realizaron tres clasificaciones para jerarquizar las temporadas de lluvia: la primera, consideró los datos históricos de precipitación, la temporada de secas (meses de febrero a mayo), lluvias (de junio a septiembre) y post lluvias (de octubre a enero); la segunda, una distinción entre los años más secos de los más lluviosos, tomando en cuenta una precipitación anual acumulada mayor de 1,000 mm; y la tercera, para obtener datos más precisos de la influencia de la precipitación pluvial en la comunidad de 
pequeños roedores, se consideró los meses más lluviosos, reacomodándolos en los meses secos ( 2 a 4 meses), lluviosos (5 a 6 meses) y poslluvias ( 3 a 4 meses).

Todas las correlaciones que se realizaron fueron para los 18 años, considerando las tres clasificaciones por las tres temporadas, $\mathrm{y}$ en los dos tipos de vegetación (Lizasoain y Joaristi, 2003).

Para calcular la diversidad beta, que es una medida de la similitud o reemplazamiento de las especies entre hábitats, se midió la tasa de recambio de las especies cuando pasan de un hábitat a otro (Koleff, 2005; Magurran, 1988; Moreno, 2001; Polo 2008). Se utilizaron dos métodos para la similitud de especies: el cuantitativo (Índice de Morisita-Horn y el Coeficiente de Similitud de Sorensen) el primer índice está fuertemente influenciado por la riqueza de especies y por el tamaño de las muestras que son altamente sensibles a la abundancia de las especies y el segundo considera la abundancia más baja de cada una de las especies compartidas en ambos sitios (Magurran, 1988; Polo, 2008). Y finalmente, el método cualitativo (Jaccard y Czekanovski-Dice-Sørensen) que consideran a las especies compartidas en ambos sitios (Magurran, 2004; Moreno, 2001).

\section{RESULTADOS}

\section{Composición de especies}

Se capturaron 13 especies de pequeños mamíferos, las cuales fueron: un marsupial (Tlacuatzin canescens), un insectívoro (Megasorex gigas), un carnívoro
(Spilogale pygmaea) y 10 roedores (Liomys pictus, Oryzomys melanotis, $O$. couesi, Nyctomys sumichrasti, Reithrodontomys fulvescens, Osgoodomys banderanus, Peromyscus perfulvus, Baiomys musculus, Sigmodon mascotensis y Xenomys nelsoni).

La curva acumulativa de especies mostró que el $53.84 \%$ de los años presentaron un total de 13 especies mientras que en el resto de años se encontró entre 6 y 12 especies que corresponden al 46.15\%. A partir de 1998 ya no se registraron más especies nuevas (Figura 2).

\section{Variabilidad temporal y espacial}

Durante los 18 años de muestreo en las selvas secas de la costa de Jalisco. Se registraron los datos de precipitación de cada uno de los años. Cabe mencionar que durante este periodo se presentaron tres fenómenos climáticos de El Niño durante los años 1992-1993 (1393.62 mm y $1213.35 \mathrm{~mm}$ ), 1998-1999 (1 $291.52 \mathrm{~mm}$ y 1 $162.31 \mathrm{~mm}$ ) y 2006-2007 (1 $059.42 \mathrm{~mm}$ y 1 $043.714 \mathrm{~mm}$ ). Esta cantidad de lluvia no influyó considerablemente en las abundancias totales, sin embargo, en las abundancias por especie sí es evidente la disminución de los individuos, para cada uno de los tipos de vegetación (Cuadro 1).

En los datos anuales,se obtuvieron la abundancia por individuos, riqueza, diversidad, diversidad máxima y equitatividad, mostrando sus valores máximos y mínimos, de igual manera para los dos tipos de vegetación; mostrando 


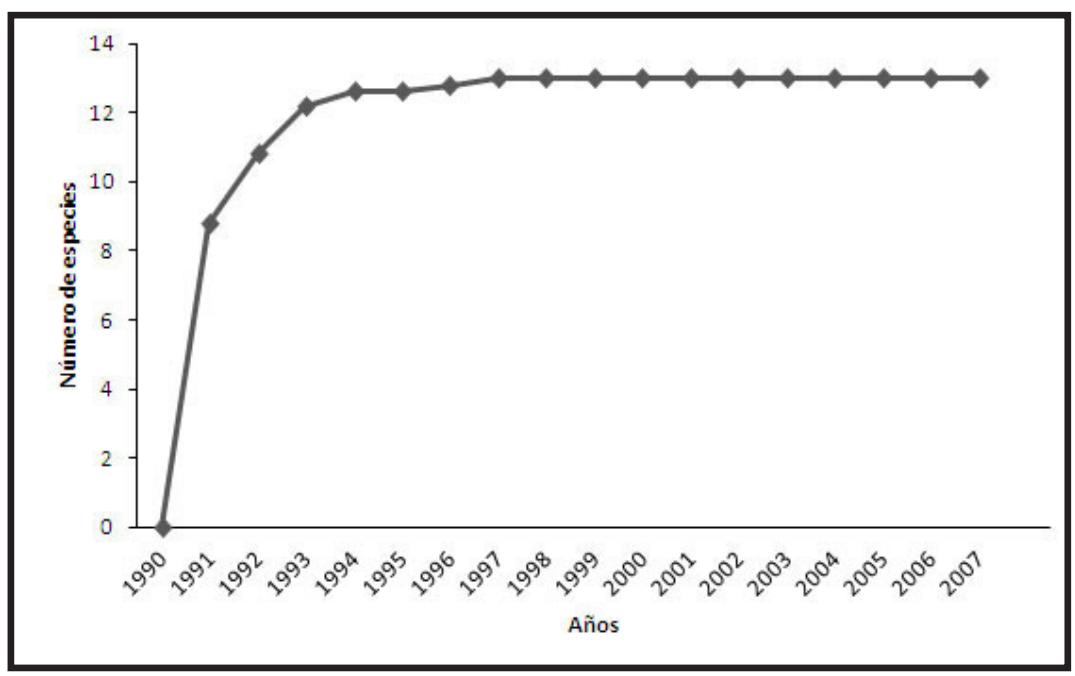

Figura 2. Curva acumulativa de las especies de pequeños mamíferos que integran la comunidad en Chamela, Jalisco.

que los valores más altos se obtuvieron en la selva mediana (Cuadro 2). Respecto a las abundancias promedio por tipo de vegetación, la selva baja presentó en promedio 486.77 individuos con un rango de 74 a 913 individuos y en la selva mediana un promedio de 381.66 individuos con un rango de 88 a 712 individuos. Por temporadas, en secas el valor máximo de individuos fue 732 con un promedio de 91.5 individuos en 2004. En lluvias se presentó un máximo de 623 individuos con un promedio de 77.8 y en post lluvias un máximo de 407 individuos con un promedio de 67.8, ambas temporadas para 1990.

Con respecto a la riqueza, los valores anuales fueron de 12 a 6 especies, el máximo de especies se encontró por primera vez en 1995, por lo que para ese año ya se habían registrado las 13 especies en las dos selvas. En selva baja se registraron hasta 10 especies y en selva mediana 12. La especie que presentó un mayor número de individuos fue Liomys pictus y esta se registró más en selva baja que en selva mediana. Las mayores diversidades se encuentran en la selva mediana, ya que en este tipo de vegetación se registraron al mayor número de especies. Tanto la riqueza de especies como la diversidad presentaron los mayores promedios en la selva mediana y en la temporada de secas (Cuadro 3). La prueba $t$ de Hutchinson mostró diferencias significativas entre los dos tipos de selvas, evidenciando que la diversidad en la selva baja es diferente a la diversidad en selva mediana durante en cada uno de los años. 
Cuadro 1. Abundancia anual, riqueza de especies y precipitacion pluvial anual, para los años en los que ocurrió el Fenómeno de El Niño, en Chamela Jalisco. Las letras indican el tipo de vegetación (b) selva baja y (m) selva mediana.

\begin{tabular}{|c|c|c|c|c|c|c|}
\hline $\begin{array}{l}\text { Años / } \\
\text { Especies }\end{array}$ & 1992 & 1993 & 1998 & 1999 & 2006 & 2007 \\
\hline \multirow[t]{2}{*}{ Baiomys musculus } & 0 & 0 & 0 & 0 & 0 & $0 \quad$ (b) \\
\hline & 0 & 3 & 0 & 0 & 2 & $4 \quad(\mathrm{~m})$ \\
\hline \multirow[t]{2}{*}{ Liomys pictus } & 696 & 131 & 612 & 54 & 168 & 495 \\
\hline & 311 & 240 & 232 & 173 & 61 & 205 \\
\hline \multirow[t]{2}{*}{ Tlacuatzin canescens } & 17 & 4 & 1 & 2 & 0 & 1 \\
\hline & 1 & 16 & 0 & 0 & 2 & 3 \\
\hline \multirow[t]{2}{*}{ Megasorex gigas } & 0 & 0 & 0 & 0 & 0 & 0 \\
\hline & 1 & 0 & 0 & 0 & 0 & 0 \\
\hline \multirow{2}{*}{ Nyctomys sumichrasti } & 3 & 0 & 0 & 0 & 0 & 0 \\
\hline & 14 & 23 & 17 & 3 & 0 & 0 \\
\hline \multirow[t]{2}{*}{ Oryzomyz melanotis } & 0 & 0 & 9 & 3 & 0 & 0 \\
\hline & 7 & 37 & 32 & 24 & 0 & 16 \\
\hline \multirow[t]{2}{*}{ Oryzomys couesi } & 0 & 0 & 4 & 5 & 0 & 0 \\
\hline & 3 & 1 & 27 & 36 & 0 & 1 \\
\hline \multirow[t]{2}{*}{ Osgoodomys banderanus } & 3 & 6 & 22 & 8 & 0 & 0 \\
\hline & 47 & 73 & 59 & 83 & 16 & 48 \\
\hline \multirow[t]{2}{*}{ Peromyscus perfulvus } & 0 & 4 & 0 & 1 & 0 & 0 \\
\hline & 9 & 51 & 56 & 38 & 6 & 27 \\
\hline \multirow[t]{2}{*}{ Reithrodontomys fulvescens } & 13 & 49 & 1 & 0 & 0 & 0 \\
\hline & 4 & 5 & 0 & 0 & 1 & 5 \\
\hline \multirow[t]{2}{*}{ Sigmodon mascotensis } & 0 & 0 & 2 & 0 & 0 & 0 \\
\hline & 0 & 0 & 0 & 0 & 0 & 1 \\
\hline \multirow[t]{2}{*}{ Spilogale pygmaea } & 1 & 0 & 0 & 0 & 0 & 1 \\
\hline & 1 & 1 & 0 & 0 & 0 & 0 \\
\hline \multirow[t]{2}{*}{ Xenomys nelsoni } & 0 & 0 & 1 & 1 & 0 & 0 \\
\hline & 0 & 0 & 1 & 0 & 0 & 0 \\
\hline \multirow[t]{2}{*}{ abundancia total } & 733 & 194 & 652 & 74 & 168 & $497 \quad$ (b) \\
\hline & 398 & 450 & 424 & 357 & 88 & $310(\mathrm{~m})$ \\
\hline \multirow[t]{2}{*}{ riqueza } & 6 & 5 & 8 & 7 & 1 & (b) \\
\hline & 10 & 10 & 7 & 6 & 6 & (m) \\
\hline $\begin{array}{l}\text { Precipitación } \\
\text { pluvial anual (mm) }\end{array}$ & 1393.62 & 1213.35 & 1291.52 & 2 1162.31 & 1059.42 & 21043.714 \\
\hline
\end{tabular}


Cuadro 2. Abundancia, riqueza, diversidad, equitatividad, diversidad máxima y precipitación anual. Datos anuales y por tipo de vegetación. Con sus valores máximos, mínimos y promedios. Los valores máximos en negritas.

\begin{tabular}{|c|c|c|c|c|c|}
\hline \multicolumn{6}{|c|}{ Datos anuales } \\
\hline \multirow[b]{2}{*}{ Abundancia } & \multicolumn{2}{|c|}{ Máximos (año) } & \multicolumn{2}{|c|}{ Mínimos (año) } & \multirow{2}{*}{$\begin{array}{l}\text { Promedios } \\
907.444\end{array}$} \\
\hline & 1625 & (1990) & 256 & $(2007)$ & \\
\hline Riqueza & 12 & $\begin{array}{c}(1995 \\
\text { y 2005) }\end{array}$ & 6 & (2007) & 9.278 \\
\hline Diversidad (H’) & & 21 (1993) & & $9(2007)$ & 0.392 \\
\hline Equitatividad (J) & & 21 (1993) & & 5 (1992) & 0.960 \\
\hline Diversidad max (H’ max) & & 79 (1995) & & $\begin{array}{l}8 \text { (2001, } \\
2002 y \text { 2007) }\end{array}$ & 0.402 \\
\hline
\end{tabular}

Selva Baja

\begin{tabular}{lcrcrrr} 
Abundancia & 913 & $(1990)$ & 74 & $(1999)$ & 486.778 \\
Riqueza & 10 & $(1996)$ & 1 & $(2006)$ & 6.278 \\
Diversidad (H') & $0.433(1999)$ & $0.013(2007)$ & 0.166 \\
Equitatividad (J) & 1 & $(1996)$ & 0 & $(2006)$ & 0.751 \\
Diversidad max (H' max) & \multicolumn{2}{c}{$0.547(1993)$} & $0.026(2007)$ & 0.199
\end{tabular}

Selva Mediana

\begin{tabular}{lccccr} 
Abundancia & 712 & $(1990)$ & 88 & $(2006)$ & 381.667 \\
Riqueza & $\mathbf{1 2}$ & $(1995)$ & 4 & $(2001)$ & 8.333 \\
Diversidad (H') & $\mathbf{0 . 8 2 1}(1991)$ & $0.365(2000)$ & 0.578 \\
Equitatividad (J) & $0.831(2003)$ & $0.368(1992)$ & 0.891 \\
Diversidad max (H' max) & $1.079(1995)$ & $0.602(2001)$ & 0.649 \\
\hline Precipitacion anual (mm) & $\mathbf{1 3 9 3 . 6 2 ( 1 9 9 2 )}$ & $383.79(2005)$ & 818.910
\end{tabular}

\section{Clasificaciones por precipitación}

En general, para todas las correlaciones de Spearman, la diversidad, la diversidad máxima y la riqueza estuvieron influenciadas por la precipitación promedio y por la precipitación anual acumulada. En especifico, para la primera clasificación de datos históricos, la selva mediana $\left(\mathrm{r}^{2}=-0.464, \mathrm{n}=54, \mathrm{P}=0.000\right)$ y la temporada de post lluvias $\left(\mathrm{r}^{2}=0.471, \mathrm{n}=\right.$ $36, \mathrm{P}=0.003)$. En los años más secos, la temporada de post lluvias $\left(\mathrm{r}^{2}=-0.533, \mathrm{n}=\right.$ $36, \mathrm{P}=0.000)$ y años más lluviosos $\left(\mathrm{r}^{2}=\right.$ $0.609, \mathrm{n}=18, \mathrm{P}=0.007)$, en ambos la 
Cuadro 3. Máximos, mínimos y promedio de la riqueza y la diversidad por tipo de vegetación y temporadas. Los valores máximos se presentan en negritas.

\begin{tabular}{lcccccc}
\hline & \multicolumn{3}{c}{ Tipo de vegetación } & \multicolumn{2}{c}{ Temporadas } \\
& Anual & $\begin{array}{c}\text { Selva } \\
\text { baja }\end{array}$ & $\begin{array}{c}\text { Selva } \\
\text { mediana }\end{array}$ & Secas & Lluvias Poslluvias \\
& & & & & & \\
\hline Riqueza & $6-12$ & $1-10$ & $4-12$ & $1-11$ & $1-9$ & $1-10$ \\
\hline Min. -Máx. & 9.27 & 6.27 & $\mathbf{8 . 3 3}$ & $\mathbf{3 . 0 4}$ & 2.24 & 2.46 \\
Promedio & & & & & & \\
\hline Diversidad & & & & & & \\
\hline Min. & 0.199 & 0.013 & 0.365 & 0.022 & 0.017 & 0.031 \\
Máx. & 0.621 & 0.433 & 0.821 & 0.815 & 0.656 & 0.709 \\
Promedio & 0.392 & 0.166 & $\mathbf{0 . 5 7 8}$ & $\mathbf{0 . 3 7 0}$ & 0.278 & 0.364 \\
\hline
\end{tabular}

diversidad fue la afectada. Para los meses más lluviosos, en la selva mediana, la diversidad $\left(\mathrm{r}^{2}=-0.488, \mathrm{n}=55, \mathrm{P}=0.000\right) \mathrm{y}$ tanto en los meses secos como también en la selva mediana, fue la diversidad máxima $\left(\mathrm{r}^{2}=-0.411, \mathrm{n}=55, \mathrm{P}=0.001\right)$.

De acuerdo a las tres clasificaciones considerando el efecto de la precipitación pluvias en la comunidad de pequeños mamíferos se encontró que la abundancia, riqueza y diversidad de los datos generales, como los promedios de estas tres variables para las tres clasificaciones fue similar, es decir, la abundancia fue mayor en la selva baja que en la selva mediana; y en el caso de la riqueza y la diversidad fueron mayores en la selva mediana que en la selva baja. Asimismo, la precipitación en la temporada de lluvias fue la que presentó los valores más altos (Figura 3, Figura 4; Cuadro 4).

Con respecto a los datos de similitud por el método cualitativo, el índice de Jaccard $\left(\mathrm{I}_{\mathrm{J}}=0.923\right)$ nos indica que ambos tipos de vegetación comparte las mismas especies, claro con excepción de Megasorex gigas que sólo se registró en la selva mediana. Para el coeficiente de similitud de Sorensen $\left(\mathrm{I}_{\mathrm{S}}=0.96\right)$ el valor fue casi cercano a 1, indicando igualmente que ambos sitios comparten las mismas especies de pequeños mamíferos.

En el caso del método cuantitativo, el índice de Morisita-Horn $\left(\mathrm{I}_{\mathrm{M}-\mathrm{H}}=0.850\right)$ el cual estuvo influenciado con la abundancia de las especies, que en este caso la especie que predomino en ambos sitios fue Liomys pictus. Para el caso del 


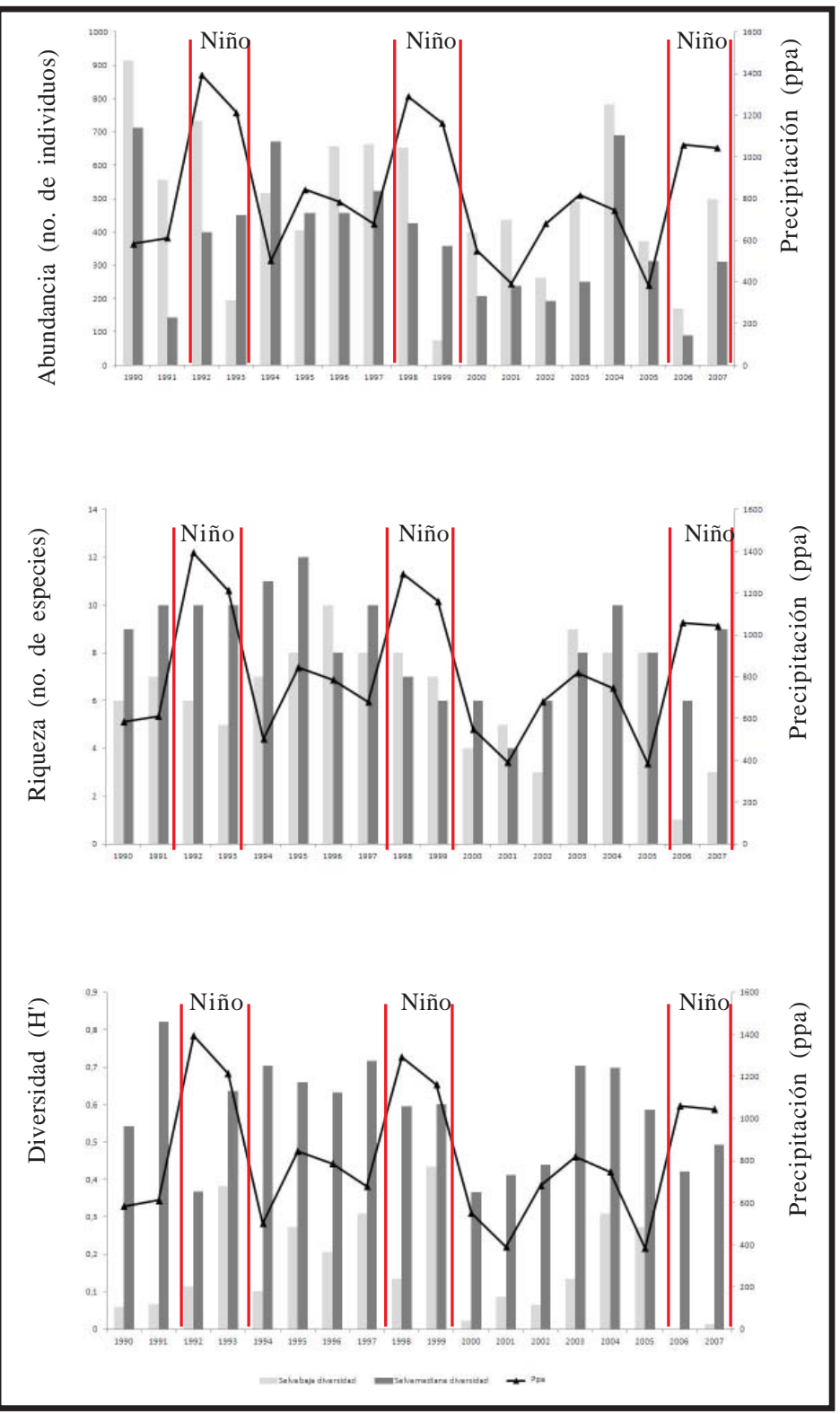

Figura 3. Datos generales de abundancia, riqueza y diversidad de los pequeños mamíferos para los dos tipos de vegetación, en Chamela, Jalisco. Entre las líneas rojas se muestran los años en que ocurrió el Fenómeno de El Niño. Las barras gis claro son selva baja, las gris oscuro la selva mediana y la linea negra la precipitación pluvial acumulada. 

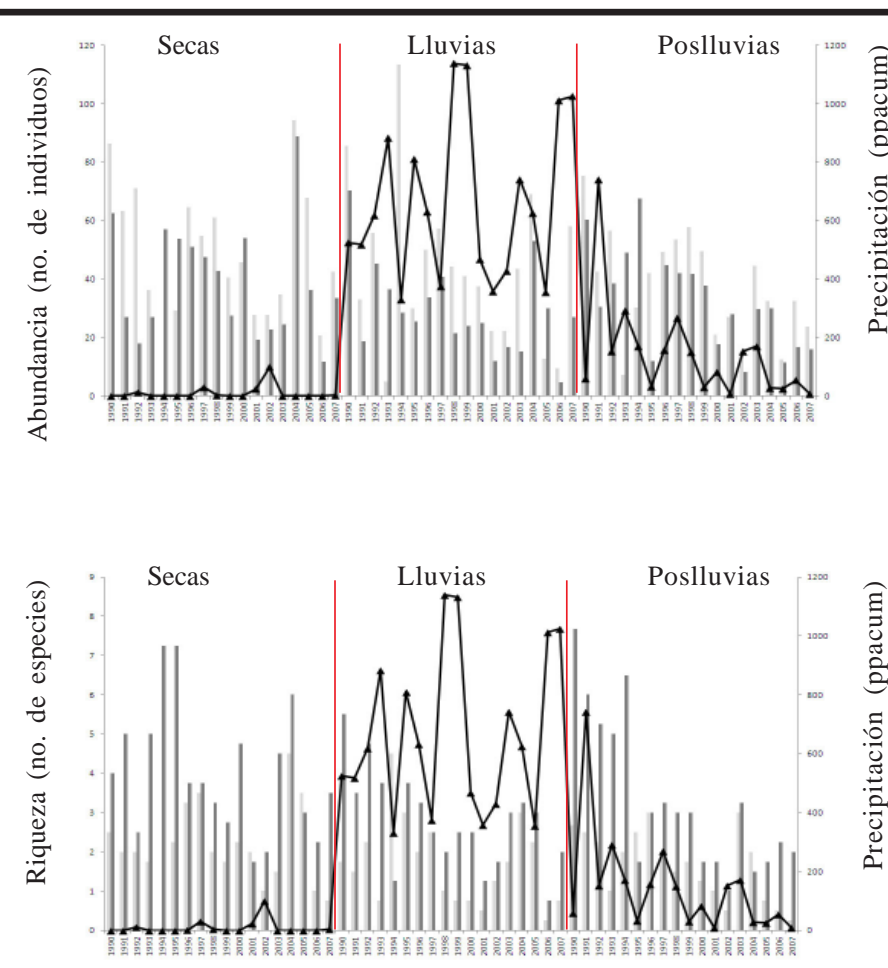

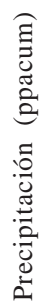

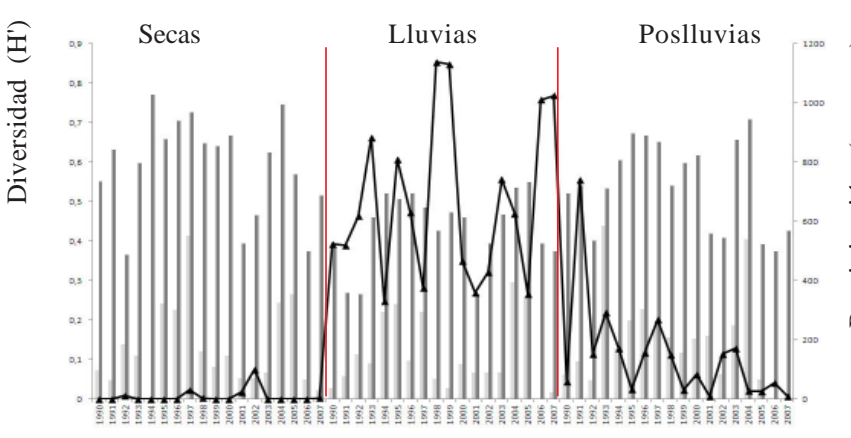

Figura 4. Datos históricos de abundancia, riqueza y diversidad de los pequeños mamíferos para los dos tipos de vegetación, en Chamela, Jalisco. Entre las líneas rojas se muestran las tres temporadas. Las barras gis claro son selva baja, las gris oscuro la selva mediana y la linea negra la precipitación pluvial acumulada. 

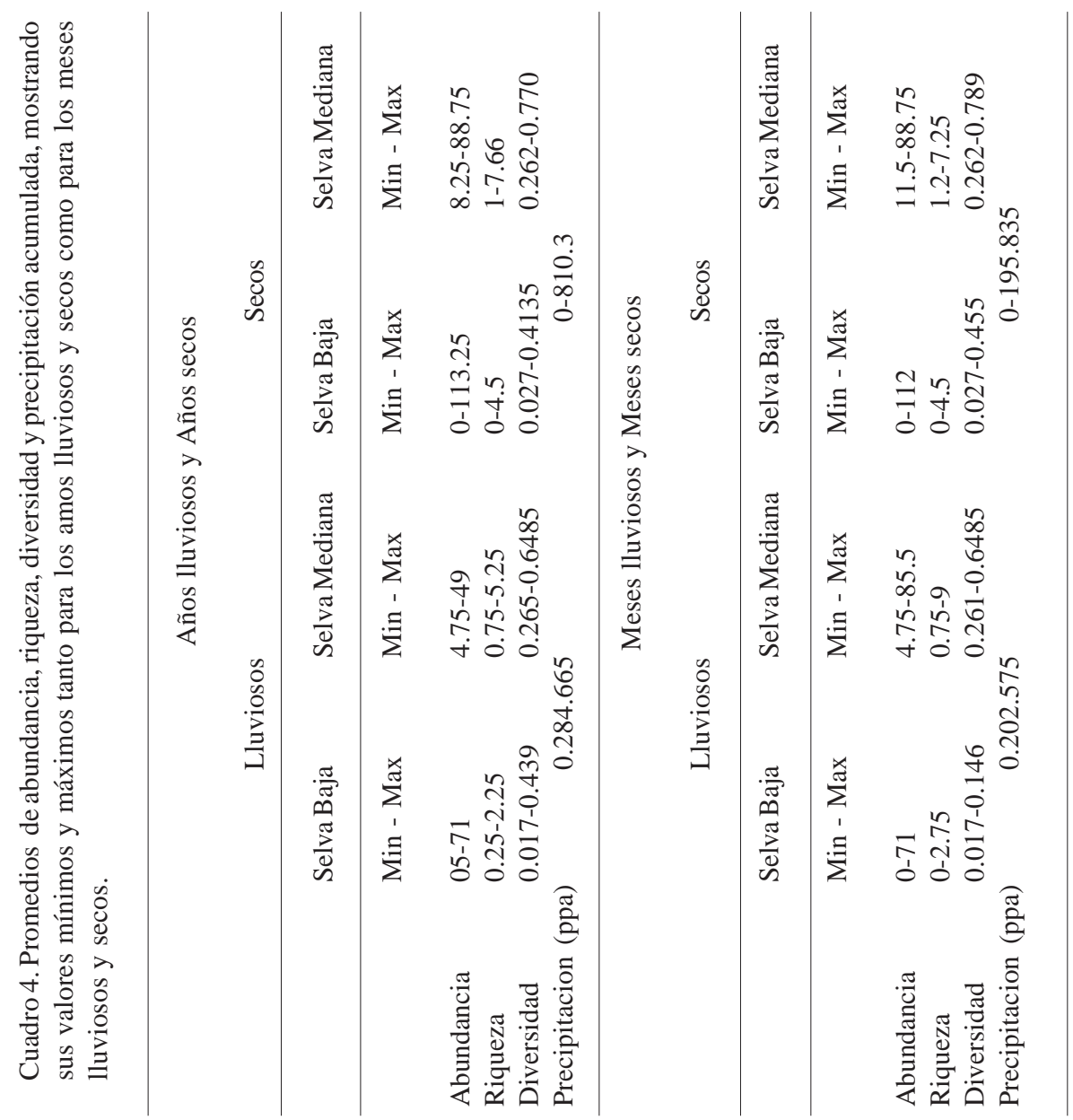


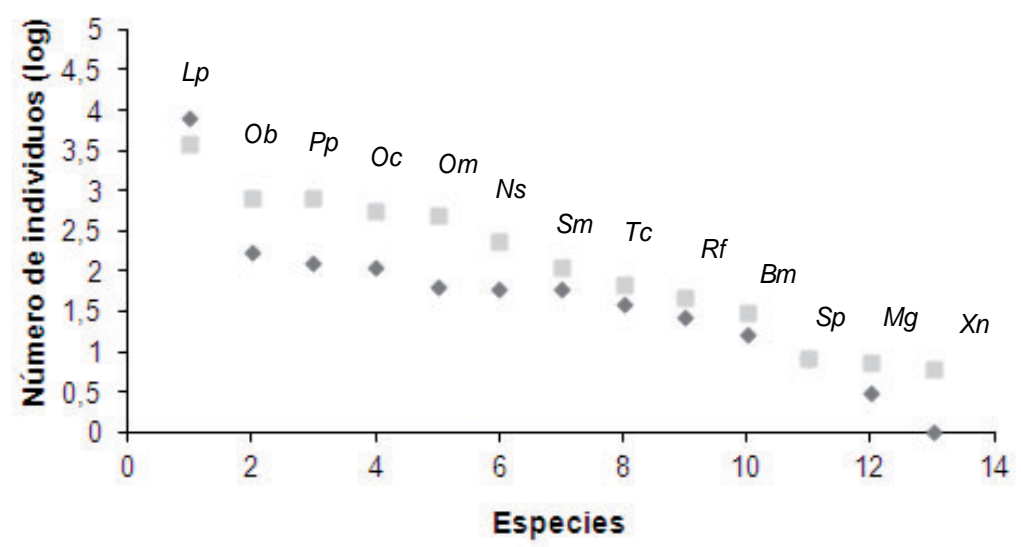

- selva baja inselva mediana

Figura 5. Comparación de las 13 especies de pequeños mamíferos en los dos tipos de vegetación. Especies ordenadas de mayor a menor abundancia. Liomys pictus (Lp), Osgoodomys banderanus (Pb), Peromyscus perfulvus (Pp), Oryzomys melanotis $(\mathrm{Om})$, Reithrodontomys fulvescens (Rf), Sigmodon mascotensis (Sm), O. couesi (Oc), Nyctomys sumichrasti (Ns), Tlacuatzin canescens (Tc), Baiomys musculus (Mm), Xenomys nelsoni (Xn), Spilogale pygmaea (Sp), Megasorex gigas (Mg).

coeficiente de Sorensen para datos cuantitativos $\left(\mathrm{I}_{\text {SCUANT }}=0.004\right)$ fue el único índice que no muestra similitud entre las especies.

En el recambio de especies por hábitats, se observó que $L$. pictus fue la especie más abundante para las dos selvas (75.56\%), principalmente en la selva baja, a diferencia de B. musculus, $M$. gigas, $N$. sumichrasti, S. pygmaea y $X$. nelsoni que fueron las especies raras (24.43\%), para ambos sitios. Sin embargo, la similitud de especies obtenida de la muestra nos indica que la composición de ambas selvas es igual (Figura 5).
Por otra parte, de las 13 especies registradas, cinco son endémicas de la región de Chamela. La mayoría de éstas se hallaron en la selva mediana (11 especies) representando el $84.6 \%$ y las especies restantes (2 especies) el $15.4 \%$ se localizaron en la selva baja. De todas las especies, la más abundante fue Liomys pictus, las comunes Orizomys couesi, Orizomys melanotis, Osgoodomys banderanus y Peromyscus perfulvus, no tan comunes Nyctomys sumichrasti, Sigmodon mascotensis y las raras Reithrodontomys fulvescens, Baiomys musculus, Tlacuatzin canescens, 
Megasorex gigas, Spilogale pygmaea y Xenomys nelsoni.

Finalmente, en cuanto al número de especies y de individuos, por tipo de vegetación, anualmente no presentaron una distribución normal. No se encontraron diferencias significativas al comparar la abundancia de individuos y el número de especies por tipo de vegetación.

\section{DISCUSIÓN}

Durante los 18 años de muestreo capturamos 13 especies de pequeños mamíferos. Ceballos en 1989, reportó 13 especies en el sitio, con diferencia de algunas como la tuza (Pappogeomys bulleri), la ardilla (Sciurus colliaei), la rata (Hodomys alleni) indicando que en Chamela hasta el momento se han registrado 16 especies de pequeños mamíferos.

La abundancia anual, para ambas selvas, estuvo influenciada por la presencia de $L$. pictus que es la especie predominante en el sitio, más en la selva baja, que en la selva mediana. Un ejemplo claro de las especies más abundantes en los sitios son Sigmodon hispidus y Microtus ochrogaster, en las praderas, su abundancia tan alta no afecta la presencia otras especies (Brady y Slade, 2001). Asimismo, la composición de especies fue similar en ambas selvas, observándose la presencia de una especie abundante (Liomys pictus), cuatro comunes (Oryzomys couesi, Oryzomys melanotis, Osgoodomys banderanus y Peromyscus perfulvus), dos no tan comunes (Nyctomys sumichrasti y Sigmodon mascotensis) y 6 las raras (Reithrodontomys fulvescens, Baiomys musculus, Tlacuatzin canescens, Megasorex gigas, Spilogale pygmaea y Xenomys nelsoni).

Se observó que al registrarse años más lluviosos la abundancia disminuyó y cuando se presentaron años muy secos la abundancia aumentó. Al presentarse dos fenómenos del Niño, éstos afectaron notablemente la abundancia y la riqueza de las especies. Estos fenómenos pueden estar asociados a la presencia de patrones cíclicos de precipitación y de sequía, ya que al ocurrir lluvias muy abundantes o periodos largos de sequía, se presentan disminuciones o extinciones locales de algunas especies (Brown y Ernest, 2002; Milstead et al., 2007; Stenseth et al., 2002). Al menos para Chamela se observó que la presencia de dos años continuos de precipitación que antecedían a cuatro o cinco años de sequía incrementaron la abundancia de individuos y de especies.

Chamela es un sitio altamente productivo, con una variedad de recursos disponibles que favorece a las especies para que habiten varios estratos de la selva, más en la selva mediana que en la selva baja, promoviendo el incremento del número de individuos por especie (Bullock y Solís-Magallanes, 1990; Ceballos, 1989, 1990; Ceballos y Valenzuela, 2010; Collet et al., 1975; Towsend et al., 2000). Es por eso que la diversidad anual fue alta en la selva mediana que en la selva baja, a diferencia de que en la primera se resguarda un mayor número de especies. La riqueza de 
especies en la temporada seca, para la selva baja fue menor que en la selva mediana, ya que en esta última, no es muy afectada por la sequía y los árboles no pierden del todo sus hojas por lo que funciona como un refugio para numerosas especies animales (Ceballos y Valenzuela, 2010; Gillesberg y Carey, 1991; Letnic y Drickman, 2005; Meserve, 1977; Viveiros, 2003; Wells et al., 2004).

De acuerdo a las tres clasificaciones para la precipitación (datos históricos, años lluviosos y años secos, meses más lluviosos) se encontró que para todos los casos la abundancia de los individuos fue mayor para la selva baja y que la riqueza como la diversidad de especies fue mayor en la selva mediana. En diversos estudios se ha demostrado que al haber una variación espacial y temporal de la precipitación, no sólo se influye directamente en la abundancia de las especies sino también a la disponibilidad de los recursos, al haber uno a dos años secos seguidos de sequía, la disponibilidad de alimento disminuye y de forma contraria al haber una mayor precipitación, la vegetación se recupera y permita la disponibilidad de recursos generando que la abundancia de las especies se mantenga o incremente (Holmgren, et al., 2006; Letnic et al., 2004; Letnic et al., 2005; Reed et al., 2006; Yarnell et al., 2007).

De las 13 especies registradas por este trabajo en Chamela, 11 son las que encuentran en la selva mediana y dos en la selva baja. L. pictus fue la más abundante en la selva baja que en la selva mediana aunque esta especie comparte el sitio con otras. La selva mediana al ser un sitio con mayor disponibilidad de agua en las partes profundas del suelo permite que las plantas con hojas en la temporada seca se mantuvieran más tiempo, haciendo que la temporada seca fuera menos marcada y que muchas especies se concentren ahí, en la temporada seca (Bullock, 1986; Ceballos y García, 1995; Ceballos y Valenzuela, 2010).

Esto se debe a que el incremento de la productividad y la complejidad estructural del hábitat, con una mayor cantidad de recursos generan un refugio, en la temporada seca, para un gran número de especies y no sólo de pequeños mamíferos sino de otros grupos de vertebrados (August, 1983; Lott et al., 1987; Ochoa, 2000; Pardini, 2004; Pardini et al., 2005; Viveiros, 2003 y Wells et al., 2004). Además, al haber un incremento en los niveles de productividad primaria, los recursos son abundantes y se obtiene un aumento en la estructura y cobertura vegetal permitiendo así la permanencia y coexistencia de los miembros de la comunidad (Brady y Slade, 2001).

\section{CONCLUSIONES}

Las selvas secas por tener una distribución restringida, ser las menos estudiadas y por albergar a un gran número de especies de vertebrados, endémicos o de distribución geográfica restringida y por ser una de las comunidades vegetales menos estudiadas adquieren una gran importancia biológica. La creación de reservas en este tipo de vegetación permitirá el desarrollo de 
estudios a largo plazo, para conocer el desarrollo de las comunidades tanto de plantas como de animales.

Por otra parte, los estudios a largo plazo permiten conocer qué ocurre en la abundancia de las especies en escalas de tiempo cortos o largos y observar como las especies se dispersan, o recolonizan otros sitios.

Finalmente, al existir sitios con una variación en recursos disponibles en diferentes estratos o niveles, hace que las especies con capacidades fisiológicas y morfológicas específicas no compitan por los recursos, sino que coexistan tanto en la selva baja como en la selva mediana. Es así que la selva mediana registrada en Chamela, presenta una mayor riqueza y diversidad de especies de pequeños mamíferos.

\section{AGRADECIMIENTOS}

Primeramente, agradecemos el apoyo financiero para la realización del trabajo a CONACyT, DGEP y Fundación Telmex. Asimismo, por su colaboración en el trabajo de campo a Ángeles Mendoza Durán y Beatriz Hernández Meza, y a Cuauhtémoc Chávez Tovar por su asesoría en el análisis estadístico.

\section{LITERATURACITADA}

August, P.V. 1983. The role of habitat complexity and heterogeneity in structuring tropical mammals communities. Ecology, 64:1495-1507.

Brandy, M.J. and N.A., Slade. 2001. Diversity of a grassland rodent community at varying temporal scales: the role of ecologically dominant species. Journal of Mammalogy, 82:974-983.

Brown, J.H. and E.J. Heske. 1990. Temporal changes in a Chihuahuan desert rodent community. Oikos, 59:290-302.

Brown, J.H. and S.K.M. Ernest. 2002. Rain and rodents: Complex dynamics of desert consumers. Biocience, 52:979-987.

Brown, J.H., Whitham, T.G., Ernest, S.K.M. and C.A. Gehring. 2001. Complex species interactions and the dynamics of the ecological systems: Long-Term experiments. Science, 293:643-650.

Bullock, S.H.1986. Climate of the Chamela Jalisco and trends in south coastal region of Mexico. Archives for Meteorology, Geophysics, and Bioclimatology, Series B, 36:297-316.

Bullock, S.H. and J. Solís-Magallanes. 1990. Phenology of canopy trees of a tropical deciduous forest in Mexico. Biotropica, 22:22-35.

Castellanos, A.E., Mooney, H.A., Bullock, S.H., Jones C. and R. Robichaux. 1989. Leaf, stem, and metamer characteristics of vines in a tropical deciduous forest in Jalisco, Mexico. Biotropica, 21:41-49.

Ceballos, G. 1989. Population and community ecology of small mammal from tropical deciduous and arroyo forest in western Mexico. Unpublished Ph. D. Thesis, University of Arizona, Tucson, Arizona.

Ceballos, G. 1990. Comparative natural history of small mammals from tropical forest in western Mexico. Journal of Mammalogy, 71:263-266.

Ceballos, G. 1995. Vertebrate diversity, ecology, and conservation in neotropical dry forests. Pp. 195-220, in: Tropical Deciduous Forest (Bullock, S., Medina E., and H. Mooney, eds.). Cambridge University Press, Cambridge. 
Ceballos, G. and A. García. 1995. Conserving neotropical biodiversity: the role of dry forest in western Mexico. Conservation Biology, 9:1349-1356.

Ceballos, G. y A. García. 2010. ChamelaCuixmala, Jalisco y Colima. Pp. 441- 446, en: Diversidad, amenazas y áreas prioritarias para la conservación de las selvas secas del Pacífico de México. (Ceballos, G., Martínez, L., García, A., Espinoza, E., Bezaury, J. y R. Dirzo, coords.) FCE - CONABIO - CONANPWWF-MÉXICO - ECOCIENCIA S.C. TELMEX.

Ceballos, G. y L. Martínez. 2010. Mamíferos. Pp. 119 - 144, en: Diversidad, amenazas y áreas prioritarias para la conservación de las selvas secas del Pacífico de México. (Ceballos, G., Martínez, L., García, A., Espinoza, E., Bezaury, J. y R. Dirzo, coords.)FCE - CONABIO - CONANPWWF-MÉXICO - ECOCIENCIA S.C. TELMEX.

Ceballos, G. y A. Miranda. 1986. Los Mamíferos de Chamela, Jalisco. Instituto de Ecología, UNAM. México, D.F.

Ceballos, G. y A. Miranda. 2000. Guía de campo de los Mamíferos de la Costa de Jalisco, México. Fundación Ecológica de Cuixmala, A.C. Instituto de Ecología e Instituto de Biología, UNAM. México, D.F.

Ceballos, G. y D. Valenzuela. 2010. Diversidad, ecología y conservación de los vertebrados de Latinoamérica. Pp. 93-118, en: Diversidad, amenazas y áreas prioritarias para la conservación de las selvas secas del Pacífico de México. (Ceballos, G., Martínez, L., García, A., Espinoza, E., Bezaury, J. y R. Dirzo, coords.) FCE - CONABIO - CONANPWWF-MÉXICO - ECOCIENCIA S.C. TELMEX.
Ceballos, G., Zsekeli, A., García, A., Rodríguez, P. y F. Noguera. 1999. Programa de Manejo de la Reserva de la Biosfera Chamela-Cuixmala. Instituto de Ecología, SEMARNAP, México, D.F.

Diffendorfer, J.E., Holt, R.D., Slade, N.A. and M.S. Gaines. 1996. Small mammal community patterns in old field. Pp. 421466, in: Long-Term Studies of Vertebrate Communities. (Cody, M.L. and J.A. Smallwood, eds.).Academic Press Inc.

Gillesberg, A.M. and A.B. Carey. 1991. Arboreal nests of Phenacomys longicaudus in Oregon. Journal of Mammalogy, 72:784-787.

Holmgren, M., Stapp, P., Dickman, C.R., Gracia, C., Graham, S., Gutierrez, J.R., Hice, C., Jaksic, F., Kelt, D.A., Letnic, M., Lima, M., López, B.C., Meserve, P.L., Milstead, W.B., Polis, G.A., Previtali, M.A., Richter, M., Sabaté, S.and F.A. Squeo. 2006. Extreme climatic events shape arid and semiarid ecosystems. Frontiers in Ecology and the Environment, 4:87-95.

Krebs, Ch.J. 1985. Ecología - Estudio de la Distribución y Abundancia. Harla. México, D.F.

Koleff, P. 2005. Conceptos y medidas de la diversidad beta. Pp. 19-40, en: Sobre diversidad biológica: El significado de las diversidades alfa, beta y gamma. (Halffter, G., Soberón, J., Koleff, P. y A. Melic, eds.).SEA, CONABIO, Zaragoza.

Kuypers, W. 2001. Statistica 6.0 STATSOFT, INC.IBM Amsterdam.

Lekve, K., Boulinier, T., Stenseth, N. Chs., Gjøsæter, J., Fromentin, J., Hines J.E. and J.D. Nichols. 2002. Spatiotemporal dynamics of species richness in coastal fish communities. Proceedings of the Royal Society of London, Series B, 269:1781-1789. 
Letnic, M. and C.R. Dickman. 2005. The responses of small mammals to patches regenerating after fire and rainfall in the Simpson Desert, central Australia. Austral Ecology, 30:24-39.

Letnic, M., Tamayo, B. and C.R. Dickman. 2005. The responses of mammals to $\mathrm{La}$ Niña (El Niño southern oscillation)associated rainfall, predation, and wildfire in Central Australia. Journal of Mammalogy, 86:689-703.

Letnic, M., Dickman, C.R., Tischler, M.K., Tamayo, B. and C.-L. Beh. 2004. The responses of small mammals and lizards to post-fire succession and rainfall in arid Australia. Journal of Arid Environments, 59:85-114.

Lima, M., Keymer, J.E. and F.M. Jaksic. 1999. El Nino-southern oscillation-driven rainfall variability and delayed density dependence cause rodent outbreaks in western South America: Linking demography and population dynamics. American Naturalist, 153:476-491.

Lizasoain, L. y L. Joaristi2003. Gestión y análisis de datos con SPSS versión 11. Thompson, Learning. Australia.

Lott, E.J. 1985. Listados Florísticos de México III. La Estación de Biología Chamela, Instituto de Biología, UNAM

Lott, E. y T.H., Atkinson. 2010. Diversidad florística. Pp. 63- 76, en: Diversidad, amenazas y áreas prioritarias para la conservación de las selvas secas del Pacífico de México. (Ceballos, G., Martínez, L., García, A., Espinoza, E., Bezaury, J. y R. Dirzo, coords.) FCE CONABIO - CONANP- WWFMÉXICO - ECOCIENCIA S.C. TELMEX.

Lott, E.J., Bullock, S.H. and A. SolísMagallanes. 1987. Floristic diversity and structure of upland and arroyo forests of Coastal Jalisco. Biotropica, 19:228-235.
Magurran, A.E. 1988. Ecological diversity and its measurements. Princeton University Press, New Jersey.

Magurran, A.E.2004. Measuring biology diversity. Blackwell Science, Ltd. Oxford, UK.

Martínez-Yrizar, A., Mass, J.M., PérezJiménez L.A. and J. Sarukhan. 1996. Net productivity of a tropical deciduous forest ecosystem in western Mexico. Journal of Tropical Ecology, 12:169-175.

M’Closkey, R.T. 1972. Temporal changes in populations and species diversity in a California rodents community. Journal of Mammalogy, 53:657-676.

Mendoza, D.M.A. 1997. Efecto de la adición de alimento en la dinámica de poblaciones y estructura de comunidades de pequeños mamíferos en un bosque caducifolio. Facultad de ciencias. Tesis de Maestría. Instituto de Ecología, UNAM.

Meserve, P.L. 1977. Three dimensional home ranges of cricetid rodents. Journal of Mammalogy, 58:549-558.

Milstead, W.B., Meserve, P.L., Ampanella, A., Previtali, M.A., Kelt, D.A. and J.R. Gutiérrez. 2007. Spatial ecology of small mammals in North-central Chile: role of precipitation and refuges. Journal of Mammalogy, 88:1532-1538.

Moreno, C.E. 2001. Métodos para medir la biodiversidad. M\&T-Manuales y Tesis SEA, Vol. 1. Zaragoza, España.

Neil McAleece, P.J., Lambshead, D. and G.L.J. Paterson. 1997. Biodiversity Pro: Free Statistics Software for Ecology. Version 2. The Natural History Museum, London. Disponible en: < http:// g c m d. na s a.gov/re cords/ NHML_Biopro.html>

Ochoa, G.J. 2000. Efectos de la Extracción de Maderas sobre la Diversidad de Mamíferos Pequeños en Bosques de 
Tierras Bajas de la Guayana Venezolana. Biotropica, 32:146-164.

Owen, J.G. 1990. Patterns of mammalian species richness in relation to temperature, productivity and variance in elevation. Journal of Mammalogy, 71:1-13.

Pardini, R. 2004. Effects of forest fragmentation on small mammals in an Atlantic Forest landscape. Biodiversity and Conservation, 13:2567-2586.

Pardini, P., Marques de Souza, S., Braga-Neto, R.and J.P. Metzger. 2005. The role of forest structure, fragment size and corridors in maintaining small mammal abundance and diversity in an Atlantic forest landscape. Biological Conservation, 124:253-266.

Polo, U.C. 2008. Índices más comunes en biología. Segunda parte, similaridad y riqueza beta y gama. Revista Facultad de Ciencias Básicas, 4:135-142.

Reed, A.W., Kaufman, G.A. and D.W. Kaufman. 2006. Species richnessproductivity relationship for small mammals along a desert-grassland continuum: differential responses of functional groups. Journal of Mammalogy, 87:777-783.

Stenseth, N. Chr., Mysterud, A., Ottersen, G., Hurrell, J.W., Chan, K. and M. Lima. 2002. Ecological effects of climate fluctuations. Science, 297:1292-1296.

Trejo, I. 2010. Las selvas secas del Pacífico mexicano. Pp. 41- 52, en: Diversidad, amenazas y áreas prioritarias para la conservación de las selvas secas del Pacífico de México. (Ceballos, G., Martínez, L., García, A., Espinoza, E., Bezaury, J. y R. Dirzo, coords.) FCE CONABIO - CONANP- WWFMÉXICO - ECOCIENCIA S.C. TELMEX.
Townsend, C.R., Harper, J.L. and M. Begon. 2000. Essentials of Ecology. Blackwell Science, Inc. Massachusetts.

Viveiros, C.E. 2003. Forest structure and vertical stratification of small mammals in a secondary Atlantic Forest, Southeastern Brazil. Studies on Neotropical Fauna and Environment, 38:81-85.

Watkins, A.J. and J.B. Wilson. 1994. Plant community structure and its relation to the vertical complexity of communities: dominance/diversity and spatial rank consistency. Oikos, 70:91-98.

Wells, K., Pfeiffer, M., Lakim, M.B. and K.E. Linsenmair. 2004. Use arboreal and terrestrial space by a small mammal community in a tropical rain forest in Borneo, Malaysia. Journal of Biogeography, 31:641-652.

Wolda, H. 1988. Seasonality and the community. Pp. 69-95, in: Organization of Communities (Gee, J.H. and P.S. Giller, eds.) Blackwell Scientific Publications, Oxford.

Yarnell, R.W., Scott, D.M., Chimimba, C.T. and D.J. Metcalfe. 2007. Untangling the roles of fire, grazing and rainfall on small mammal communities in grassland ecosystems. Oecologia, 154:387-402.

Zar, J.H. 1996. Biostatistical analysis. Tercera edición. Prentice Hall. New Jersey. 